\title{
Réponses à Marta Caravà, Jean-Marie Chevalier et Roberta Dreon
}

\section{Pierre Steiner}

\section{(2) OpenEdition \\ 1 Journals}

Édition électronique

URL : http://journals.openedition.org/ejpap/1953

DOI : 10.4000/ejpap.1953

ISSN : 2036-4091

Éditeur

Associazione Pragma

\section{Référence électronique}

Pierre Steiner, « Réponses à Marta Caravà, Jean-Marie Chevalier et Roberta Dreon », European Journal of Pragmatism and American Philosophy [En ligne], XII-1 | 2020, mis en ligne le 16 juin 2020, consulté le 10 décembre 2020. URL : http://journals.openedition.org/ejpap/1953; DOI : https://doi.org/10.4000/ ejpap.1953

Ce document a été généré automatiquement le 10 décembre 2020.

\section{(1) (9)}

Author retains copyright and grants the European Journal of Pragmatism and American Philosophy right of first publication with the work simultaneously licensed under a Creative Commons AttributionNonCommercial-NoDerivatives 4.0 International License. 


\title{
Réponses à Marta Caravà, Jean- Marie Chevalier et Roberta Dreon
}

\author{
Pierre Steiner
}

Marta Caravà synthétise de manière très juste et claire les ambitions de la lecture que je propose de Wittgenstein dans le troisième chapitre de l'ouvrage: il s'agit de penser d'une manière critique renouvelée le projet des sciences cognitives, en particulier dans leurs versions énactives ou énactivistes. Avant de répondre aux questions légitimes qu'elle m'adresse, je voudrais dissiper un malentendu concernant le statut de ce que j'appelle, à la suite de Wittgenstein, les "concepts psychologiques." Dans son texte, Marta Caravà m'attribue l'idée que les concepts psychologiques en tant que concepts de la psychologie ne doivent pas être compris comme des concepts référentiels, c'est-à-dire comme des concepts dont l'usage premier consisterait à référer à ou à dénoter des entités, structures ou processus. Ces concepts permettent au contraire de qualifier des comportements, à partir de "normes épistémiques définies dans et par une pratique de recherche" (je la cite). J'adhère globalement à un tel instrumentalisme sur le sens des concepts scientifiques (même s'il faut aménager, au sein de la pluralité des usages normés des concepts scientifiques, une place pour les usages ponctuellement référentiels); il rejoint d'ailleurs l'inférentialisme que je défends dans le chapitre II de l'ouvrage. Mais lorsque je parle, dans le chapitre III, des concepts psychologiques, je fais avant tout référence, avec Wittgenstein, aux concepts ordinaires que nous mobilisons quotidiennement dans nos explications ou interprétations du comportement d'autrui. Les concepts psychologiques ne sont pas d'abord et principalement les concepts forgés et utilisés dans les laboratoires de psychologie. Ce n'est donc pas un point de vue scientifique, mais l'usage de ces concepts psychologiques ordinaires qui contribue à faire d'un comportement un comportement expressif d'une vie mentale. Avant d'étudier ce qu'il appelle "vision," le psychologue mobilise et connaît déjà le concept psychologique ordinaire de "voir." L'étude scientifique de la vision ne consiste pas, ici, à préciser le référent du concept ordinaire de "voir," car ce dernier concept n'est ni théorique ni référentiel. La psychologie de la vision étudie les processus et mécanismes qui sous-tendent causalement la production et le maintien d'un comportement que nous appelons "voir" à partir de différents critères. Pour étudier et modéliser ces 
processus, le psychologue peut produire de nouveaux concepts ("module," "liage perceptif," "attention," "représentation"), ici des concepts psychologiques parce que propres à la psychologie, mais il serait erroné de penser que ces concepts de laboratoire ont le même sens et les mêmes usages que les concepts psychologiques ordinaires. Ces derniers, pour Wittgenstein, peuvent d'ailleurs constituer un obstacle à la pratique de la psychologie scientifique: ${ }^{1}$ 'est aussi pour cela que ces deux types de concepts psychologiques doivent être soigneusement distingués.

2 Cette précision étant faite, je peux aborder la première question posée par Marta Caravà: critiquer l'énactivisme parce qu'il n'aurait pas renoncé à l'idée que la cognition serait un processus générique dont les différentes déclinaisons sont dénotées par les concepts psychologiques en général, n'est-ce pas là une disqualification philosophique hâtive d'un projet de recherche scientifiquement prometteur? Si, après avoir rejeté des concepts et images classiques de la cognition comme le représentationnalisme, l'internalisme ou l'individualisme, on en vient à jeter aussi par-dessus bord le modèle du processus pour comprendre la cognition, que reste-t-il? En quoi d'ailleurs une adhésion à cette image du processus serait-elle incompatible avec le déploiement d'une véritable alternative scientifique aux théories cognitives classiques? La question est tout à fait légitime. L'énactivisme qui est discuté et critiqué dans l'ouvrage est avant tout une théorie philosophique de la cognition, qui se réclame d'une radicalité conceptuelle: les succès empiriques qui sont invoqués (en robotique, psychologie de la vision, neurosciences de l'action, ...) ont rarement été générés par la posture philosophique elle-même. Il s'agit plutôt, pour l'énactivisme, de proposer une interprétation nouvelle et heureuse de résultats empiriques déjà existants. Dans ce sens, je suis assez réservé sur les conséquences empiriques directes et originales qu'aurait pour l'instant cette théorie philosophique de la cognition. Pour discuter, apprécier et critiquer cette théorie, il s'agit de rester sur le terrain de la philosophie, en questionnant sa cohérence et sa radicalité effective. Plus généralement, c'est sur ce terrain que les comparaisons et confrontations entre différentes traditions peuvent être clarifiées, voire avancées. Par exemple, on associe souvent aux sciences cognitives " $4 \mathrm{E}$ " (embodied, embedded, enactive, extended) une redécouverte de la phénoménologie, phénoménologie qui nourrirait la radicalité de ces approches $4 \mathrm{E}$ : il serait pourtant malheureux de limiter la situation actuelle à une confrontation entre une philosophie analytique caricaturée et une phénoménologie libératrice (vers l'action!, vers l'expérience!, vers l'intentionnalité non-représentationnelle!). Ici aussi, il est important de valoriser l'originalité et l'exigence du pragmatisme (et du pragmatisme wittgensteinien) par rapport à ces deux traditions.

3 Cela étant, la deuxième question de Marta Caravà s'impose également: concrètement, d'un point de vue opératoire, quelles sont les conséquences sur le plan de la recherche cognitive de l'approche expressiviste que je défends à partir de Wittgenstein? Cette approche ne prétend pas promouvoir une nouvelle manière de pratiquer une recherche empirique, au-delà de préceptes méthodologiques externalistes, nonreprésentationnalistes et anti-individualistes déjà progressivement adoptés dans différentes disciplines, préceptes qui ne sont donc pas le monopole du pragmatisme, fût-il wittgensteinien. La différence spécifique faite par la conception expressiviste est notamment la suivante: abandonner le modèle du processus pour étudier la cognition, c'est notamment inviter les théories cognitives putativement "radicales" à s'émanciper d'une métaphysique naturaliste naïve, qui suppose par exemple que "cognition" 
correspond à un ensemble spécifique de processus naturels, qui posséderaient une essence ou un trait commun ("la marque du cognitif") indépendamment de nos conventions et pratiques descriptives. Cela ne signifie pas que "la" cognition n'existe pas, mais plutôt que sa reconnaissance dans un système précède et excède l'étude de sa réalisation matérielle, qu'elle soit neurocentrée, énactée, ou étendue. Qu'est-ce à dire exactement? Je vais préciser cette réponse à Marta Caravà en répondant également à Roberta Dreon et à Jean-Marie Chevalier, qui m'interrogent, dans leurs textes respectifs, sur les effets d'une autre thèse défendue dans le chapitre II de l'ouvrage, et avec laquelle la thèse expressiviste est en continuité. Selon cette thèse défendue dans le chapitre II, le cerveau fonctionne comme un muscle: ${ }^{2}$ il conditionne nos performances comportementales (guidage, motricité, attention, ...), mais c'est à partir de ces performances - et non pas de la contribution du cerveau à lui seul - que les épisodes mentaux sont attribués ou attribuables, dans un cadre inférentialiste et expressiviste. Les travaux de Thomas Fuchs (2012) (en psychiatrie), de Lambros Malafouris (2019) (en archéologie cognitive), ou d'Ezequiel Di Paolo \& Hanne De Jaegher (2012) et Shaun Gallagher (2018) (dans le champ de la cognition sociale) défendent eux aussi cette thèse du cerveau comme muscle. Ajouter à cette thèse la thèse expressiviste sur les concepts psychologiques et la cognition, c'est aller plus loin. Le cerveau possède et exerce des capacités plastiques de réponse à l'environnement, qu'il importe évidemment de comprendre (en forgeant notamment de nouveaux concepts psychologiques); mais il faut aussi - et surtout, dirais-je - comprendre comment ces réponses peuvent s'intégrer à et constituer le répertoire comportemental historiquement, culturellement et anthropologiquement situé à partir duquel les concepts psychologiques ordinaires sont appris et utilisés. L'adverbialisme et l'expressivisme ouvrent cette voie de recherche, en allant donc plus loin que la reconnaissance d'une distribution ou extension factuelle de l'esprit et de la cognition sur un ensemble de dispositifs, de capacités et d'événements (dont le cerveau comme muscle).

Il m'est impossible de réagir ici aux nombreux points soulevés par Jean-Marie Chevalier dans son texte riche et stimulant. Étant donné que certaines des questions qu'il m'adresse croisent des questions aussi exprimées par Marta Caravà et Roberta Dreon, je vais ici traiter de quelques points abordés spécifiquement par Jean-Marie Chevalier.

5 Esprit, intérieur et extérieur. Jean-Marie Chevalier questionne la valeur des arguments inférentialistes et wittgensteiniens proposés pour délocaliser l'esprit au-delà de la dualité peu éclairante entre "intérieur" et "extérieur." Ma stratégie lui paraît reposer sur quelques confusions ou raccourcis, dont le suivant: ce n'est pas parce que les conditions d'individuation et d'attribution des significations et concepts ne sont pas intracrâniennes mais sociales que les épisodes de pensée eux-mêmes, ou du moins leurs conditions de possibilité, ne seraient pas dans la tête. Pour citer Jean-Marie Chevalier, "ce n'est pas parce que la pensée a besoin de conditions institutionnelles pour se développer qu'il n'en demeure rien en tant que processus interne," mais toute la question est justement de savoir ce qui pourrait demeurer dans le crâne de manière telle que ce reste puisse réaliser un épisode de pensée. Une pétition de principe prend place si l'on considère a priori que les épisodes de pensée sont des événements intracrâniens, à charge pour l'externaliste de montrer le contraire. Il est alors facile de critiquer l'externalisme au nom d'une distinction de principe initiale entre ce qu'est la pensée, et 
ses conditions sociales d'individuation et de développement. L'approche pragmatiste que je développe dans l'ouvrage, en particulier à partir de Brandom et de Wittgenstein, renverse la perspective: comment des événements intracrâniens pourraient-ils réaliser, même partiellement, des épisodes de pensée? Avons-nous d'ailleurs besoin d'une telle logique de réalisation? L'approche déontologique considère qu'il n'est plus nécessaire de poser ici un véhicule matériel (interne ou externe) qui transporterait (comment, d'ailleurs?) des contenus de pensée conceptuels. Mais, objecte Jean-Marie Chevalier, ne peut-il pas y avoir des épisodes de sensation ou d'émotion indépendamment de tout contexte et de toute expression comportementale? Ne peut-on pas par exemple imaginer l'occurrence d'une expérience volitive, affective ou émotionnelle chez un sujet à la suite d'une manipulation directe de son tissu cérébral par un savant malintentionné? Qu'un sujet puisse ressentir quelque chose de nouveau, d'inattendu ou d'inédit à la suite d'une telle stimulation n'est pas ici en question. Mais une sensation peut-elle à elle seule constituer une expérience de joie, de plaisir ou encore de volonté? Les différences entre ces expériences sont-elles seulement ou même principalement des différences sensorielles? Il serait facile et décevant d'invoquer ici des conventions linguistiques qui détermineraient la spécificité mentale de chaque sensation. Mais on peut en revanche s'intéresser aux capacités qui amèneraient le sujet (ou ceux qui l'observent) à qualifier ce qu'il (le sujet) ressent en utilisant de manière préférentielle certains concepts comme "joie" ou "plaisir." Comment avons-nous appris à utiliser le concept de "joie"? Je ne reviendrai pas ici sur les apories d'une réponse dénotationnelle, qui associe au concept une expérience par principe privée. Le concept de "joie" est acquis et utilisé pour qualifier un ensemble ouvert de trames comportementales possibles et attendues. Si quelqu'un peut être joyeux en ressentant quelque chose, c'est parce que cette sensation s'articule de jure à ces trames comportementales (linguistiques et motrices) qui constituent également les critères d'usage du concept "joie." La situation imaginée par Jean-Marie Chevalier peut donc être décrite d'au moins deux manières différentes: on peut penser que la stimulation cause non pas seulement une sensation mais un état de joie, de peine, ou de volonté, car elle modifie une structure matérielle qui devient - par le fait de cette intervention, et quelque peu magiquement - la base de réalisation de la joie, peine, volonté... Mais, alternativement - et c'est évidemment la voie privilégiée par l'adverbialisme et par l'expressivisme que je tire de Wittgenstein - on peut soutenir que la stimulation cause une sensation (en modifiant une structure matérielle), sensation qui n'est que l'élément initial d'une trame comportementale exprimant la joie, la peine ou la volonté dépendamment de sa qualification par des agents (y compris le sujet de l'expérience) qui maitrisent les concepts de "joie," "peine," ou "douleur." Sans sensation causée par stimulation, il n'y aurait probablement pas d'expérience, mais cela ne signifie pas que l'expérience soit identifiable à la sensation. De même, "l'effet que cela fait de voir rouge" requiert plus qu'une sensation de rouge, sans pour autant nécessiter un jugement réflexif: cela nécessite la maîtrise du concept de rouge et (donc) un ensemble de pratiques inférentielles partagées. ${ }^{3}$ Ici aussi, l'intentionnalité de l'expérience (voir rouge, et non pas voir vert) n'est pas un fait interne. Une stratégie semblable peut être adoptée pour rendre compte de ce que l'on appelle "connaissance à la première personne," ou "autorité en première personne": ces phénomènes peuvent s'expliquer sans partir de - ou arriver à - l'hypothèse qu'il existerait des faits mentaux internes qui seraient les objets d'une connaissance échappant aux réquisits publics de la connaissance tout court. ${ }^{4}$ 
6 Esprit, adverbe et dispositions. J'accorde à Jean-Marie Chevalier qu'il serait pour le moins étrange de penser se débarrasser des immeubles en n'utilisant plus le mot "immeuble," et en décrivant les immeubles uniquement par un ensemble de fonctions. En tant qu'entités matérielles (survenant sur des points d'espace-temps), les immeubles existent et ne se réduisent pas aux manifestations et fonctions qu'ils rendent en fait possibles. Ce constat pourrait aussi s'appliquer à l'esprit... si ce dernier était une entité matérielle. Or, c'est justement ce point fondamental qui est questionné dans l'ouvrage, en particulier à partir de l'ontologie adverbiale de Dewey, dont la présentation tardive (Steiner 2019: 188-94) ne doit pas dissimuler l'importance méthodologique qu'elle a revêtue pour moi (l'imperfection de l'exposition de ce cheminement m'incombe évidemment): pour simplifier, c'est à partir de cette ontologie que j'ai été amené à (re)lire Wittgenstein et Brandom. Dans ce cadre, l'esprit n'est pas une substance, ce n'est pas une activité (spirituelle ou comportementale) ou une disposition à l'action, c'est un adverbe ou, pour le dire encore autrement, une manière d'agir. Cette manière d'agir peut être décrite par un nombre ouvert d'adverbes ("avec attention," "intelligemment," "en anticipant" ...). Y a-t-il ici une réintroduction du definiendum dans le definiens, comme l'objecte Jean-Marie Chevalier? Oui, si l'on présuppose que des attitudes comme l'attention ou l'anticipation sont les effets ou les manifestations de la présence d'un esprit. Non, si ces attitudes sont elles-mêmes ramenées à des manières d'agir circonstanciées: lire avec attention, ce n'est pas lire et faire quelque chose de distinct; c'est lire d'une certaine manière, qui s'atteste dans un ensemble de gestes et de capacités. Capacités et dispositions peuvent figurer parmi les manières d'agir auxquelles l'esprit est ramené, mais il n'y a pas d'identification et encore moins de réduction de l'esprit à des dispositions.

7 Le problème corps-esprit. En tant que vocabulaire, le mentalisme possède des vertus descriptives et prédictives; il constitue plus fondamentalement une partie de l'image manifeste (Sellars) de l'homme dans le monde. Mais - et c'est là où je me sépare de Jean-Marie Chevalier - cela n'implique pas que ce vocabulaire soit en correspondance avec des faits matériels qui ne devraient rien à ce que nous faisons et disons pour être des faits "mentaux." Le problème corps-esprit, comme Rorty permet de le comprendre, apparaît lorsque l'on veut faire du vocabulaire mental une représentation d'une réalité substantielle et spécifique (phénoménale, matérielle...). Ce problème corps-esprit, dans l'ouvrage, est un repère: il s'agit d'en traiter pour le dépasser. Jean-Marie Chevalier perçoit bien l'ambivalence de la posture: en traiter, ce serait déjà le conserver, ou du moins lui faire encore trop d'honneur contrairement à la revendication initiale de dépassement vers une approche adverbialiste ou expressiviste. J'assume cette posture «bifrons» (pour reprendre l'expression de Jean-Marie Chevalier), non sans avoir conscience de son ambiguïté. Le problème corps-esprit repose sur une compréhension erronée (représentationnelle et substantielle) du vocabulaire mental et des concepts psychologiques; une approche "scientifique" ne garantit en rien que l'on neutralise ou dépasse cette compréhension fautive, au contraire. Il est ainsi important de revenir sur les multiples formes que ce problème a pu prendre tout au long de l'histoire, et sur ses différents contextes de formulation et de réapparition (y compris en sciences cognitives). Un certain pragmatisme aurait tort de croire que ce problème est derrière nous: il est l'expression maladroite et biaisée d'une perplexité réelle que nous ressentons quand nous nous interrogeons sur le mode d'existence de nos pensées, intentions, désirs et sensations, et sur le statut des concepts psychologiques. 
La question fondamentale que pose Roberta Dreon (mais aussi, d'une manière différente, Jean-Marie Chevalier) concerne l'unité du projet et du propos de l'ouvrage, et en particulier celle des auteurs mobilisés. La linéarité du parcours ne doit notamment pas dissimuler une tension importante entre, d'un côté, une approche purement grammaticale et thérapeutique (Rorty, Wittgenstein) et, de l'autre, une approche qui, en plus d'être critique, serait aussi positive et intégrative (Dewey), alliant une pars construens à une pars destruens. Cette tension apparaîtrait particulièrement lorsque l'on prend connaissance de mes lectures et usages de Dewey: Dewey propose certes une critique des approches substantialistes et intellectualistes de l'esprit, mais il n'en reste pas là. La critique d'une philosophie comme ensemble de croyances théoriques ne peut se faire qu'à partir d'un ensemble de dispositions à agir et à penser qu'il convient aussi d'assumer et de préciser: l'erreur serait de penser que l'on peut philosopher et critiquer de nulle part, et en restant nulle part (Roberta Dreon décrit bien dans sa conclusion le caractère immanent de toute enquête selon le pragmatisme). Cette tension, que je qualifierais de méta-philosophique, est parfois rapprochée par Roberta Dreon d'une autre tension, à mon sens davantage méthodologique: celle entre un pragmatisme qui réduirait l'esprit et l'expérience à un ensemble de vocabulaires et à leurs usages normatifs (qui sont bien plus que linguistiques), et un pragmatisme qui propose une histoire naturelle de l'esprit en récupérant, je la cite, "l'organique, la sensibilité, le qualitatif." En un mot, une philosophie de l'esprit qui soit aussi une philosophie de l'expérience historicisée.

9 Je partage évidemment avec Roberta Dreon l'idée que la théorie deweyienne de l'esprit est plus large que la généalogie du problème corps/esprit, la théorie de la signification, l'adverbialisme ou l'émergentisme qu'il propose et que j'exploite dans le chapitre IV. Roberta Dreon ne me reproche d'ailleurs pas d'être incomplet; ses remarques, bien plus subtiles et constructives, discutent l'architectonique que j'adopte lorsque je traite de Dewey. Dès le départ, si l'on peut parler ainsi, Dewey se situe sur un autre plan que celui des auteurs précédemment discutés, avec des conséquences importantes au niveau de sa conception de l'esprit. Je suis d'accord avec cela. Pourtant, et sans embrasser un œcuménisme facile et stérile, il n'y a pas à choisir entre la philosophie positive de l'expérience proposée par Dewey et l'analyse critique d'obédience rortyenne et wittgensteinienne. Les deux se complètent, ce qui ne signifie pas bien sûr une absence de frictions au niveau des engagements méta-philosophiques et de la question (peutêtre trop souvent dramatisée) du statut de l'expérience. Renoncer, avec Rorty et Wittgenstein, à certaines images et mythes portant sur l'esprit, c'est aussi découvrir de nouvelles manières de penser. Faire avec moins, c'est aussi pouvoir faire plus. Critiquer, c'est déjà transformer. Je dirais même que, par rapport au projet d'élaborer une théorie générale de l'esprit et de l'expérience, le travail critique permis par Rorty et Wittgenstein se rapproche davantage, par bien des aspects, de ce que Dewey appelait "enquête." Le travail critique et thérapeutique est un travail en situation: il prend pour objet des théories et débats contemporains, dont il problématise les apories. Mais pas seulement. Les assomptions dont on se débarrasse (le représentationnalisme, l'internalisme, le référentialisme) sont les effets d'une mécompréhension de nos pratiques. Rendre compte de cette mécompréhension, c'est alors mettre au jour certaines dimensions de nos pratiques, dimensions qui nous sont devenues opaques. L'histoire naturelle de l'esprit que Roberta Dreon appelle de ses vœux, à partir de 
Dewey, ne peut faire l'économie d'une analyse de nos manières de parler de l'esprit; c'est en se débarrassant de certaines de ces manières que nous pourrons aussi penser et concevoir autrement l'esprit et ses dimensions expérientielles.

Dans l'introduction de l'ouvrage (Steiner 2019: 19-20), je précise que la lecture que je propose de la conception deweyienne de la science et des techniques participe au projet d'élaborer une anthropologie philosophique globale: les pratiques au sein desquelles l'expérience et le langage se font et se transforment sont aussi des pratiques techniquement structurées et constituantes. Pour réaliser ce projet, il faut notamment questionner l'articulation proposée par Dewey entre philosophie de l'esprit et intelligence expérimentale. Ce n'est pas l'expérimentalisme technique qui peut être un repère ou un modèle de l'agir social et politique; c'est plutôt la technique comme ensemble de médiations qui devient une ressource structurante et contraignante du champ de l'expérience. La critique de l'articulation faite par Dewey entre sa philosophie de l'esprit et son projet socio-politique m'a plus occupé dans le chapitre $\mathrm{V}$ de l'ouvrage que les prolongements positifs de cette philosophie de l'esprit, pour une raison simple: cela m'a permis de problématiser davantage les reprises ou les réactualisations contemporaines de Dewey, dans un contexte historiographique ayant notamment renoncé à une vision héroïque ou discontinuiste de la science moderne. Une fois que l'on a questionné quelques présupposés des ambitions sociales et politiques de la philosophie deweyienne de l'esprit, on peut s'intéresser autrement - et positivement - aux rapports entre technique et esprit chez Dewey. La technique est à la fois un mode d'expérience et un ensemble de significations opératoires (voir Expérience et nature, chapitre 5): elle englobe et articule l'organique et le grammatical, pour reprendre le vocabulaire de Roberta Dreon. Le représentationnalisme estime que nos pratiques linguistiques et cognitives sont face à l'expérience et face au monde, et que c'est en vertu de représentations ou d'une intentionnalité "mentale" qu'elles peuvent atteindre le monde ou faire référence. À la place de la relation de représentation, de figuration, ou de visée intentionnelle, il s'agit d'insister sur les médiations - sémiotiques, normatives, techniques - dans lesquelles nos pratiques se déploient. ${ }^{5}$ C'est ici que l'anthropologie philosophique ne rencontre pas seulement les sciences cognitives, mais aussi l'histoire et les sciences sociales. Il ne s'agit alors pas, par exemple, d'ajouter au concept ou à la phrase des dispositifs d'ancrage et de fonctionnement pour qu'ils puissent atteindre le monde, mais d'en proposer une description inférentialiste et technique qui fasse l'économie de ces besoins superflus. En faire plus, ce n'est pas ajouter une entité ou un ordre nouveau de phénomènes, c'est décrire autrement l'expérience et le faire humain une fois que nous nous sommes débarrassés de fausses nécessités représentationnalistes ou internalistes. En conclusion, je pense que ces tensions méta-philosophiques et méthodologiques rappelées par Roberta Dreon peuvent être défaites, apaisées ou assumées en jugeant sur pièce, de manière circonstanciée. Si l'on souhaite débuter par une exposition des principes fondamentaux du pragmatisme que l'on prétend ensuite "appliquer," il est vraisemblable que l'on rencontrera un ensemble de dualités sédimentées (thérapie vs. (re)construction; analyse grammaticale vs. enquête empirique; expérience vs. langage), dualités dont le caractère mutuellement exclusif des éléments risque de s'imposer à nous de façon automatique. Si l'on s'efforce avant tout de faire usage du pragmatisme en problématisant, réaménageant ou dépassant certaines questions (ici: l'esprit), on n'échappera évidemment pas à la nécessité, chemin faisant, d'exprimer et de clarifier des engagements plus généraux, mais il n'est pas certain que les oppositions et dualités 
mentionnées plus haut auront entre-temps résisté au travail d'enquête qui aura été mené.

\section{BIBLIOGRAPHIE}

DEWEY John, (2008), Middle Works, Vol. 4, Carbondale, Southern Illinois University Press.

DEWEY John, (2013 [1925]), Expérience et nature, trad. Joëlle Zask, Paris, Gallimard.

DI PAOLO Ezequiel \& Hanne DE JAEGHER, (2012), “The Interactive Brain Hypothesis," Frontiers in

Human Neuroscience, 7 juin.

FUCHS Thomas, (2012), Das Gehirn - Ein Beziehungsorgan: Eine phanomenologisch-okologische

Konzeption, Stuttgart, Kohlhammer.

GALLAGHER Shaun, (2018), "Decentering the Brain: Embodied Cognition and the Critique of Neurocentrism and Narrow-Minded Philosophy of Mind," Constructivist Foundations, 14 (1), 101-34. MALAFOURIS Lambros, (2019), "Mind and Material Engagement," Phenomenology and the Cognitive Sciences, 18, 1-17.

PEIRCE Charles Sanders, (1868), “Questions Concerning Certain Faculties Claimed for Man,” Journal of Speculative Philosophy, 2, 103-14.

SELLARS Wilfrid, (1992 [1956]), Empirisme et philosophie de l'esprit, trad. F. Cayla, Combas, Éditions de l'Éclat.

STEINER Pierre, (2019), Désaturer l'esprit. Usages du pragmatisme, Paris, Questions Théoriques.

STEINER Pierre, (2020), "Habit, Meaning and Intentionality: A Deweyan Reading," in F. Caruana \&

I. Testa (eds.), Habits. Pragmatist Approaches from Cognitive Neurosciences to Social Sciences,

Cambridge University Press, à paraître.

WITTGENSTEIN Ludwig, (2004 [1953]), Recherches philosophiques, trad. F. Dastur, M. Elie, J.-L. Gautero, D. Janicaud et E. Rigal, Paris, Gallimard.

\section{NOTES}

1. Voir par exemple Recherches philosophiques, §577.

2. Cette idée se trouve déjà chez Dewey en 1908: "That the brain frees organic behavior from complete servitude to immediate physical conditions, that it makes possible the liberation of energy for remote and ever expanding ends is, indeed, a precious fact, but not one which removes the brain from the category of organic devices of behavior" (2008: 132).

3. Voir par exemple Sellars (1992).

4. Voir par exemple Peirce (1868, question 4).

5. Sur ce point, je me permets de renvoyer à mon essai (2020). 


\section{AUTEUR}

\section{PIERRE STEINER}

Université de Technologie de Compiègne, $\mathrm{COSTECH}$

Pierre.Steiner[at]utc.fr 